\title{
Association analysis of copy numbers of FC-gamma receptor genes for rheumatoid arthritis and other immune-mediated phenotypes
}

\author{
Lude Franke ${ }^{1,14}$, Hanane el Bannoudi ${ }^{2,14}$, Diahann TSL Jansen², Klaas Kok ${ }^{1}$, Gosia Trynka ${ }^{3,4,5}$, \\ Dorothee Diogo $^{3,4,5}$, Morris Swertz ${ }^{1,6}$, Karin Fransen ${ }^{1,7}$, Rachel Knevel ${ }^{2}$, Javier Gutierrez-Achury ${ }^{1}$, \\ Lisbeth Ärlestig ${ }^{8}$, Jeffrey D Greenberg ${ }^{9}$, Joel Kremer ${ }^{10}$, Dimitrios A Pappas ${ }^{11}$, Alexandros Kanterakis ${ }^{1,6}$, \\ Rinse K Weersma ${ }^{7}$, Annette HM van der Helm-van Mil ${ }^{2}$, Viktor Guryev ${ }^{12}$, Solbritt Rantapää-Dahlqvist ${ }^{8}$, \\ Peter K Gregersen $^{13}$, Robert M Plenge ${ }^{3,4,5}$, Cisca Wijmenga ${ }^{1}$, Tom W-J Huizinga ${ }^{2}$, Andreea Ioan-Facsinay ${ }^{2}$, \\ Rene EM Toes ${ }^{2,15}$ and Alexandra Zhernakova ${ }^{\star, 1,2,15}$
}

\begin{abstract}
Segmental duplications (SDs) comprise about $5 \%$ of the human genome and are enriched for immune genes. SD loci often show copy numbers variations (CNV), which are difficult to tag with genotyping methods. CNV in the Fc receptor region (FCGR) has been suggested to be associated with rheumatic diseases. The objective of this study was to delineate association of FCGR-CNV with rheumatoid arthritis (RA), coeliac disease and Inflammatory bowel disease incidence. We developed a method to accurately quantify CNV in SD loci based on the intensity values from the Immunochip platform and applied it to the FCGR locus. We determined the method's validity using three independent assays: segregation analysis in families, arrayCGH, and whole genome sequencing. Our data showed the presence of two separate CNVs in the FCGR locus. The first region encodes FCGR2A, FCGR3A and part of FCGR2C gene, the second encodes another part of FCGR2C, FCGR3B and FCGR2B. Analysis of CNV status in 4578 individuals with RA and 5457 controls indicated association of duplications in the FCGR3B gene in antibody-negative RA $(P=0.002$, OR $=1.43)$. Deletion in FCGR3B was associated with increased risk of antibody-positive RA, consistently with previous reports $(P=0.023, O R=1.23)$. A clear genotype-phenotype relationship was observed: CNV polymorphisms of the FCGR3A gene correlated to CD16A expression (encoded by FCGR3A) on CD8 T-cells. In conclusion, our method allows determining the CNV status of the FCGR locus, we identified association of CNV in FCGR3B to RA and showed a functional relationship between CNV in the FCGR3A gene and CD16A expression.

European Journal of Human Genetics (2016) 24, 263-270; doi:10.1038/ejhg.2015.95; published online 13 May 2015
\end{abstract}

\section{INTRODUCTION}

Segmental duplications (SDs) are loci with two or more, highly similar, duplicated regions. These loci cover about $5 \%$ of the human genome and are enriched for immune-mediated genes. ${ }^{1,2}$ One of the SDs encodes the low-affinity human FC-gamma receptors ( $\mathrm{F} c \gamma \mathrm{R})$. $\mathrm{F} c \gamma \mathrm{R}$ are glycoproteins that bind the $\mathrm{Fc}$ region of $\operatorname{IgG}$ and have a pivotal role in many immunological processes. ${ }^{3-8} \mathrm{Fc} \gamma \mathrm{R}$ are expressed by various immune cell types and provide a critical link between the humoral and the cellular compartments of the immune system. The proteins encoded by FCGR genes (CD16, encoded by FCGR3, and CD32, encoded by FCGR2) are cell surface receptors that are involved in the process of phagocytosis and the clearing of immune complexes.
Genetic analysis of the FCGR locus in genome-wide association studies (GWAS) has been limited, because genes encoding the Fc $\gamma \mathrm{R}$ molecules are located in highly homologous SD blocks. The low-affinity FCGR gene family is located in blocks of two repeats of $\sim 82 \mathrm{~kb}$ with $>98 \%$ being identical. It includes three FCGR2 genes (FCGR2A (CD32A), FCGR2B (CD32B) and FCGR2C (CD32C)) and two FCGR3 genes (FCGR3A (CD16A) and FCGR3B (CD16B)). The genetic structure of the low-affinity FCGR gene family in relation to blocks of SD is presented in Figure 1a-c.

Because of the complex genetic structure of the FCGR locus, only the promoter and the first three exons of the FCGR2A gene are tagged by genetic variants present on current genome-wide genotyping

\footnotetext{
${ }^{1}$ Department of Genetics, University of Groningen, University Medical Centre Groningen, Groningen, The Netherlands; ${ }^{2}$ Department of Rheumatology, Leiden University Medical Centre, Leiden, The Netherlands; ${ }^{3}$ Division of Rheumatology, Brigham and Women's Hospital, Harvard Medical School, Boston, Massachusetts, USA; ${ }^{4}$ Division of Genetics, Brigham and Women's Hospital, Harvard Medical School, Boston, Massachusetts, USA; ${ }^{5}$ Program in Medical and Population Genetics, Broad Institute of MIT and Harvard, Cambridge, Massachusetts, USA; ${ }^{6}$ Genomics Coordination Center, University of Groningen, University Medical Center Groningen, Groningen, The Netherlands; ${ }^{7}$ Department of Gastroenterology and Hepatology, University of Groningen, University Medical Centre Groningen, Groningen, The Netherlands; ${ }^{8}$ Department of Public Health and Clinical Medicine/Rheumatology, Umeå University, Umeå, Sweden; ${ }^{9}$ Department of Medicine, New York University School of Medicine, New York, New York, USA; ${ }^{10}$ Department of Medicine, Albany Medical College, Albany, New York, USA; ${ }^{11}$ Department of Medicine, Columbia University College of Physicians and Surgeons, New York, New York, USA; ${ }^{12}$ Laboratory of Genome Structure and Ageing, European Research Institute for the Biology of Ageing, University of Groningen, University Medical Centre Groningen, Groningen, The Netherlands; ${ }^{13}$ Feinstein Institute for Medical Research, Manhasset, New York, USA

${ }^{*}$ Correspondence: Dr A Zhernakova, Department of Genetics, University Medical Centre Groningen, P.0. Box 30001, Groningen 9700RB, The Netherlands. Tel: +31 50 3617100; Fax: +31 50 3617230; E-mail: a.zhernakova@umcg.nl

${ }^{14}$ These authors wish it to be known that, in their opinion, they should be regarded as joint first authors.

15These authors wish it to be known that, in their opinion, they should be regarded as joint last authors.

Received 31 July 2014; revised 27 March 2015; accepted 15 April 2015; published online 13 May 2015
} 


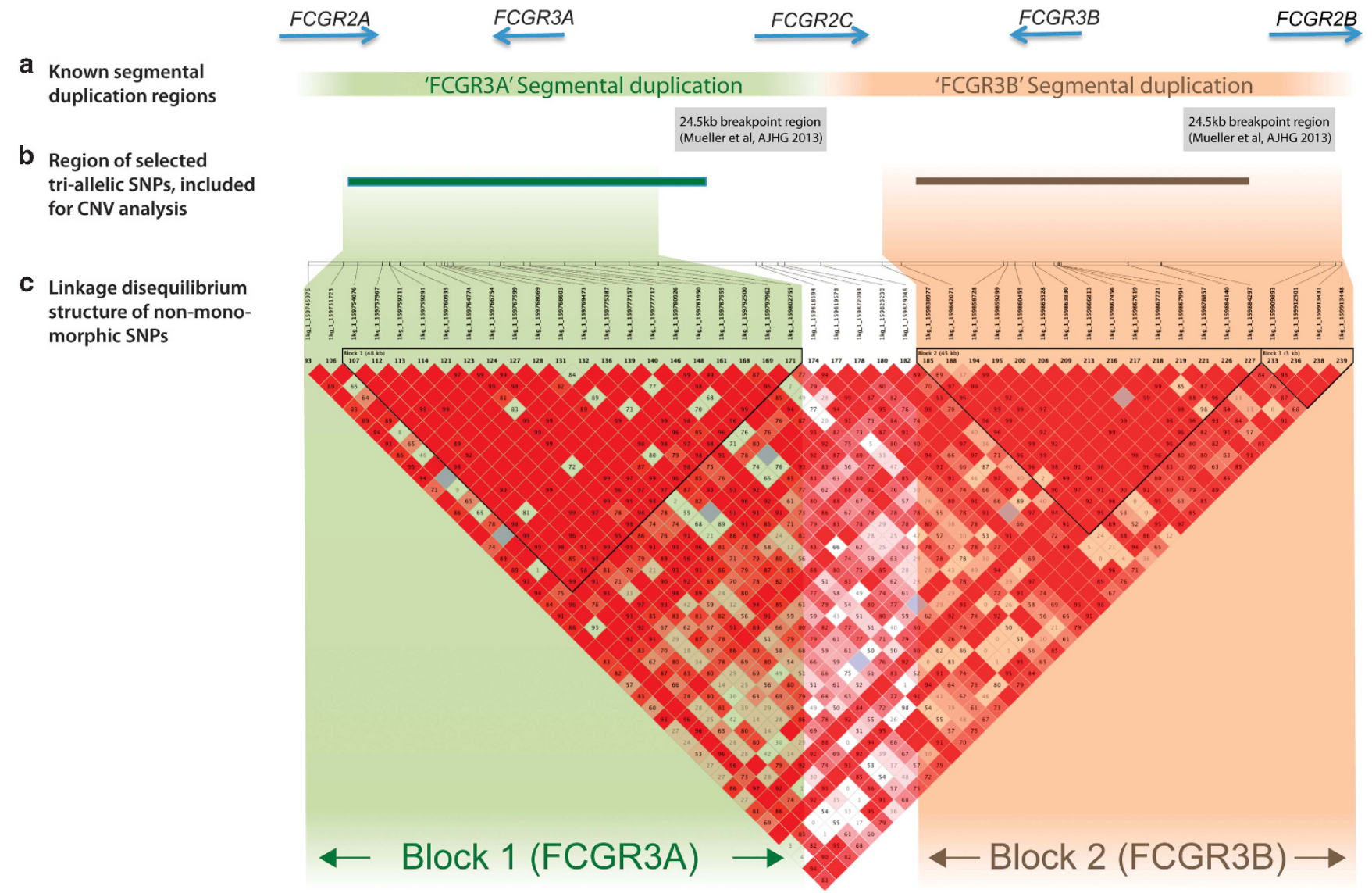

Figure 1 The genetic structure of the FCGR locus. The genetic structure of the low-affinity FCGR gene family in relation to blocks of SD and LD in the locus. The LD block is constructed based on analysis of 43 single-nucleotide polymorphisms that passed quality control and had MAF $>5 \%$ in Dutch RA and control cohorts.

platforms. SNPs in the FCGR2A gene (outside the SD locus) are strongly associated with inflammatory bowel disease (IBD) and in particular to its subgroup ulcerative colitis (UC). ${ }^{9,10}$ Less strong association was reported with systemic lupus erythematosus (SLE) $\left(\text { rs1801274:A }>\mathrm{G}, P=6.78 \times 10^{-7}\right)^{11}$ and rheumatoid arthritis (RA) (rs12746613:C $\left.>\mathrm{T}, P=2 \times 10^{-5}\right){ }^{12}$ The exact genetic risk of the whole locus contributing to disease is unknown and cannot be assessed by GWAS. However, several reports of deletions and duplications of $\mathrm{F} c \gamma \mathrm{R}$ genes have been published. ${ }^{13-18}$ The relevance of copy number variants (CNV) in the Fc $\gamma \mathrm{R}$ locus in SLE has been strongly suggested by several studies, ${ }^{4,15,18-21}$ whereas similar analyses in RA showed contradictory results. ${ }^{18}$

At least four experimental techniques have been proposed to quantify copy numbers within this SD. These include quantitative $\mathrm{PCR},{ }^{19}$ comparative genomic hybridization (arrayCGH), ${ }^{22}$ multiplex ligand-dependent probe amplification ${ }^{23}$ and several variations of paralogous ratio tests (PRT), including the use of PRT in combination with quantitative sequence variant (QSV) assay. ${ }^{17,24}$ However, none of these methods provides an accurate estimation of both the number and location of CNVs. Methods based on quantitative PCR give a continuous, rather than discrete, value of $\mathrm{CNVs}$ (reviewed in McKinney et $a l^{18}$ ). Other methods aim to design sets of primers and probes specific to the FCGR3A or FCGR3B genes, which is a complicated task given the high homology of these genes. The PRT method, including the genotyping of a reference diploid sequence on chromosome 18 , is probably the most robust reported to date, ${ }^{24}$ but it does not allow identification of CNV boundaries. Recently, the combination of a PRT probe with QSV was applied to an RA casecontrol analysis, allowing an estimation of CNV in 1115 RA patients and 654 controls. ${ }^{17}$ This study suggested that there is an association with lower $\mathrm{CNV}$ in the FCGR3B gene in RA patients.

The Immunochip is an Illumina array, which includes SNPs to fine map 186 distinct loci associated with at least 1 of the 12 immunemediated diseases, including RA, CeD and IBD., ${ }^{9,25-28}$ The low-affinity $\mathrm{F} c \gamma \mathrm{R}$ locus is also included in the Immunochip platform for finemapping. The Immunochip was not specifically designed to quantify CNVs, as its primary focus was on calling bi-allelic SNP genotypes. Previously, several methods of quantifying CNV changes based on intensity values of genotyping probes have been proposed. ${ }^{29-37}$ As these methods rely upon raw intensity measures, it is crucial to properly account for any systematic differences that may exist (eg, batch effects), which is especially important when comparing many thousands of samples that have been hybridized in different laboratories. In this study, we developed a highly robust method to accurately quantify FCGR-CNV using the Immunochip, by extensively correcting for various confounders through principal component analysis (PCA). We confirmed our results using three independent methods (segregation analysis in families, arrayCGH and nextgeneration sequencing (NGS) analysis) and then applied our method to a cohort of 4578 RA patients as well as 5235 individuals with two other immune-mediated diseases (CeD and IBD) and 7941 controls. We identified association of CNVs in FCGR3B in RA. Functional 
analysis showed that copy numbers in the FCGR locus have a clear effect on the expression of Fc $\gamma$ Rs in major blood cell types.

\section{MATERIALS AND METHODS}

Sample collection

After quality control, our sample collection included 4578 RA cases from three populations (the Netherlands, Sweden and the USA), 1477 IBD samples (900 $\mathrm{CD}$ and $577 \mathrm{UC}$, all from the Netherlands), $3758 \mathrm{CeD}$ cases from five populations (the Netherlands, Poland, Spain, Italy and India) and 7941 matched population controls. In total, 17754 samples were included in the case-control analysis. In addition, 285 offspring from the Genome of the Netherlands (GoNL, www.nlgenome.nl) ${ }^{38}$ were included for segregation analysis, giving a total of 18039 individuals (Table 1). Written informed consent was obtained from all subjects; the research was approved by the ethics committee or institutional review boards of all the participating institutions.

\section{SNP genotyping}

Samples were genotyped using the Immunochip according to Illumina's (San Diego, CA, USA) protocols at five laboratories (listed in Supplementary Methods). The final report file, including $\mathrm{R}$ intensity values, was extracted for all FCGR and reference SNPs, as listed in Supplementary Table S1.

\section{Data quality control}

The Illumina GenomeStudio GenTrain2.0 algorithm was used to cluster samples. Only samples with call rates $>99 \%$ that also passed the QC for the primary Immunochip analysis in each disease (described in Jostins et al, ${ }^{9}$ Eyre et $a^{25}$ and Trynka et $a^{26}$ ) were included. We performed an extra check to exclude any duplicates or first-degree relatives in the combined analysis.

\section{FCGR copy number count quantification}

The algorithm for CNV estimation is described in detail in the Supplementary Methods section.

\section{Identification of $\mathrm{CNVs}$ by arrayCGH}

In 22 individuals, the complete FCGR locus was assessed by a dense set of arrayCGH probes, using a custom-designed array (Agilent, Santa Clara, CA, USA; ID 029465). In total, 2704 arrayCGH probes were included in the extended $F c \gamma R$ locus, of which 1171 were located in the SD (see Supplementary Methods for further details).

\section{Sequencing analysis}

We selected 20 individuals with various combinations of CNVs in blocks 1 and 2 from the GoNL study, ${ }^{38}$ for whom, on average, $14 \mathrm{x}$ whole genome sequences were made and Immunochip SNP genotypings were available. We predicted their CNV status using a dynamic window approach (DWAC-Seq, http://tools. genomes.nl/dwac-seq.html)), see Supplementary Methods for details.

\section{Statistical analysis}

CNV quantification was performed using the customized Java software (available at https://github.com/molgenis/systemsgenetics/wiki/Copy-numberdetermination-using-ImmunoChip-intensity-data-for-the-FCGR-locus.

Association analysis was done by chi-square testing using SPSS (Armonk, NY, USA) and R. Correlation of genotypes and expression of the protein was performed in SPSS v19. Meta-analysis was performed using an inverse variance meta-analysis using R. Power calculations were performed using http://pngu. mgh.harvard.edu/ purcell/gpc/. The Breslow-Day test for genetic heterogeneity was performed using R. The haplotype analysis was done using Haploview, ${ }^{39}$ default settings.

\section{Functional studies}

To stain CD16 molecules on different cell types, fresh leukocytes were isolated from the blood of 21 healthy individuals using HetaSep (StemCell Technologies, Vancouver, BC, Canada). Cells were stained with antibodies against CD3, CD4, CD8, CD14, CD16 and CD19.

To identify which isoform of CD16 (CD16a or CD16b) was expressed on CD8+ cells, leukocytes were left untreated or were treated with $5 \mathrm{U} / \mathrm{ml}$ phosphatidylinositol-specific phospholipase C (PI-PLC) for $1 \mathrm{~h}$ at $37^{\circ} \mathrm{C}$ under constant mixing. After extensive washing, cells were stained with antibodies

Table 1 Sample collection

\begin{tabular}{lcccc}
\hline Population & Controls (\% females) & Cases (\% females) & CCPpos (\% females) & CCPneg (\% females) \\
\hline RA cohort & & & & \\
NL & $1379(44 \%)$ & $648(67 \%)$ & $332(67 \%)$ & $300(66 \%)$ \\
SW & $942(68 \%)$ & $675(68 \%)$ & $406(67 \%)$ & $160(65 \%)$ \\
US_NY & $1275(69 \%)$ & $2238(74 \%)$ & $1567(74 \%)$ & $531(76 \%)$ \\
US_B & $1861(75 \%)$ & $1017(79 \%)$ & $1006(79 \%)$ & 0 \\
Total & 5457 & 4578 & 3311 & 1617 \\
\hline
\end{tabular}

\begin{tabular}{lccr} 
Population & Controls (\% females) & Cases (\% females) & Total \\
\hline Coeliac disease & & & \\
NL & $1379(44 \%)$ & $1110(68 \%)$ & 2489 \\
Italy & $1255(81 \%)$ & $1374(67 \%)$ & 2629 \\
Poland & $532(42 \%)$ & $505(63 \%)$ & 1037 \\
Spain & $306(53 \%)$ & $545(68 \%)$ & 851 \\
India & $391(16 \%)$ & $224(55 \%)$ & 615 \\
Total & 3863 & 3758 & 7621
\end{tabular}

\begin{tabular}{lccccc}
\hline $\begin{array}{l}\text { Population } \\
\text { IBD }\end{array}$ & Controls (\% females) & IBD cases (\% females) & CrD (\% females) & UC (\% females) & Total \\
\hline $\mathrm{NL}$ & $1379(44 \%)$ & $1548(65 \%)$ & $900(65 \%)$ & $577(48 \%)$ & 2927 \\
\hline All study & Controls & Cases & Family members & All samples & \\
\hline Total & 7941 & 9813 & 285 & 18039 \\
\hline
\end{tabular}


against $\mathrm{CD} 3, \mathrm{CD} 8, \mathrm{CD} 15$ and $\mathrm{CD} 16$ - or an irrelevant isotype-matched antibody for $20 \mathrm{~min}$ at $4{ }^{\circ} \mathrm{C}$. The expression of CD16 on CD8+ T cells, and neutrophils was assessed by FACS. Detailed information is described in Supplementary Methods.

\section{Data sharing}

The results of this study are submitted to DGVArchive (http://www.ebi.ac.uk/ dgva/data-download), submission number estd222.

\section{RESULTS}

\section{Haplotype structure of the FCGR locus}

To gain insight into the haplotype structure of the FCGR locus, we first investigated the Immunochip Genome Studio cluster plots for 1159 SNPs located in the block of SD in Dutch RA cases and controls. Only 114 SNPs $(9.8 \%)$ passed our quality criteria for SNP genotyping (minor allele frequency $(\mathrm{MAF})>0.1 \%$, Hardy Weinberg equilibrium (HWE) $P$-value $>0.0001)$. Of these, 75 SNPs $(6.5 \%)$ had a MAF $>1 \%$, while only 43 SNPs (3.7\%) passed the quality criteria with a MAF $>5 \%$.

Haplotype analyses on SNPs with MAF > 5\% showed strong linkage disequilibrium (LD) across the whole locus but identified two LD blocks. The split between the two blocks $\left(D^{\prime}=0.68\right)$ corresponded approximately to the split between the two duplicated regions (Figure 1a-c). As the two CNV blocks included the complete sequence of the FCGR3A gene (left block) and the FCGR3B gene (right block), respectively, we will refer to these as the FCGR3A and FCGR3B CNV loci, although the borders of both CNVs are wider than the FCGR3A or 3B genes (see Figure $1 \mathrm{a}-\mathrm{c}$ ).

Visual inspection of all 1159 SNP clusters clearly indicated the presence of several SNPs with a CNV pattern (ie, more than three clusters were visible per SNP, see Supplementary Figure S1). In total, at least 13 SNPs with a CNV pattern were located in the FCGR3A block, and at least 11 SNPs showed a CNV pattern in the FCGR3B block (Supplementary Tables S2 and S3). Next, we developed an algorithm to estimate $\mathrm{CNV}$ in the FCGR locus based on analysing the fluorescent dye intensities of multiple SNPs genotyped on the Illumina Immunochip platform, as described in the Supplementary Methods.

We performed the analysis of CNVs in the FCGR locus in three ways: (1) All 1159 SNPs were included, indicating the average number of copies in both FCGR3A and FCGR3B blocks (Supplementary Table S1). (2) PCA of $13 \mathrm{CNV}$-like SNPs from the FCGR3A block (Supplementary Table S2). (3) PCA of 11 CNV-like SNPs from the FCGR3B block (Supplementary Table S3). In all the three analyses, the first principal component from the PCA of the FCGR SNP intensity data correlated the number of copies for each individual (Supplementary Figures S2a-c). We also investigated whether any of the first 10 PCs from the combined analysis of all 1159 FCGR SNPs reflected the CNV status of FCGR3A and/or FCGR3B blocks, and we found that the third PC does correspond to the CNV status of the FCGR3A block. Consistent with this observation, of the 100 top SNPs that explain most of the third PC, 97 are annotated in the FCGR3A block. Combining the first and third PCs from the 1159 SNP analysis showed the clearer cluster separations in all populations, concordant with the results obtained by analysing the FCGR3A and FCGR3B blocks (Figure 2). In consequence, we used this method to define the $\mathrm{CNV}$ status. In both duplicated blocks, three, four and more copies could not be undoubtfully separated from each other; they were therefore combined in one group of $\geq 3$ copies.

After estimating the CNV status for each individual, we validated our $\mathrm{CNV}$ estimation algorithm by three independent methods: segregation analysis in families, arrayCGH, and whole genome sequencing.

\section{Segregation in the families}

We first studied the segregation of the FCGR CNVs in 257 trio families to verify the outcome of our new method. For the FCGR3A block, the inferred FCGR3A CNV genotypes of the 257 trios showed perfect Mendelian inheritance, indicating that our method correctly assigned these genotypes. The same was also true for the FCGR3B block, except for two unlikely events in the distribution of FCGR3B CNV, which could occur due to recombination or uneven distribution of CNV on parental chromosomes. The genotypes of all parent-child trios are presented in Supplementary Table S4.

\section{ArrayCGH analysis}

We next used arrayCGH to confirm the CNV quantification yielded by our method. We selected 22 individuals representing three different CNVs in the FCGR3A locus (1,2 and $\geq 3$ copies), and four CNV types in FCGR3B (0, 1, 2 and $\geq 3$ copies) based on the PCA analysis. The selection and CNV genotypes of these 22 samples are indicated in Supplementary Table S5.

ArrayCGH analysis showed perfect correlation with the combined number of copies of FCGR3A+FCGR3B blocks. However, the arrayCGH method could not separate the CNVs seen in the two blocks, not even when the analysis was performed only on probes annotated as unique for either block. For example, individuals with a double deletion of the FCGR3B block showed a low signal across the whole FCGR locus (2/4 copies, Supplementary Figure S3a), while those with a single deletion of the FCGR3B or FCGR3A block showed a similar pattern of deletion on the whole FCGR block on arrayCGH analysis (3/4 copies) (Supplementary Figures S3b-c). Similarly, individuals with three copies of FCGR3A or FCGR3B showed similar arrayCGH patterns (Supplementary Figure S3d). We concluded that arrayCGH confirmed the results of our PCA analysis but that arrayCGH cannot be used to assess the CNV structure of the FCGR locus accurately.

\section{Next-generation sequencing analysis}

To further confirm our PCA-based method of estimating CNVs, we compared the results with those from NGS analysis. We selected 20 individuals with various $\mathrm{CNV}$ distributions in the FCGR3A and FCGR3B blocks and performed a CNV analysis of the whole genome sequence data using DWAC-Seq. Of the $40 \mathrm{CNV}$ s included for confirmation analysis, we observed a perfect match between the results obtained by both methods (Supplementary Table S6).

Together, the results from segregation analysis, arrayCGH and sequencing suggest that our method accurately estimates the CNV structure in the FCGR locus.

\section{Structural composition of the FCGR locus}

We next analysed the structural composition of FCGR CNVs in the cohort of 17754 individuals. The FCGR3A deletion is extremely rare: only 2 out of the 17754 individuals carried a double deletion $(0$ copies) for FCGR3A, and 275 individuals (1.5\%) harboured a deletion of one FCGR3A allele. CNV in FCGR3B is more common: we identified 46 out of the $17754(0.26 \%)$ individuals with double deletion of the FCGR3B locus, whereas 1304 subjects (7.4\%) had a deletion of one copy of the FCGR3B block. There were no individuals with a double deletion of both genes, and only 9 out of the 17754 individuals had a single-copy deletion in both the FCGR3A and FCGR3B loci. The frequency of CNV per population and in every disease group is presented in Supplementary Tables S7-S9. 


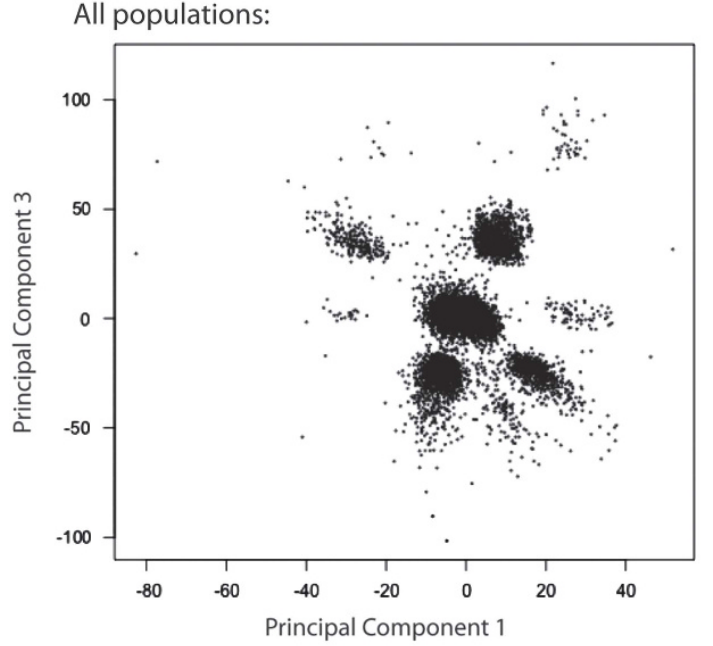

Individual populations:

Dutch population

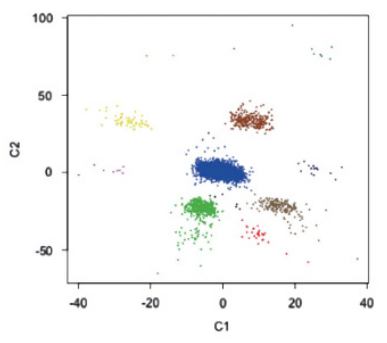

Spanish population

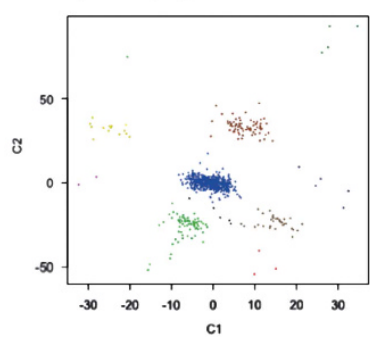

Indian population

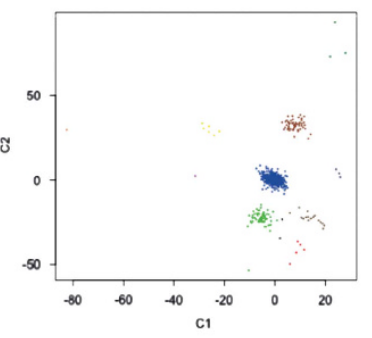

Swedish population

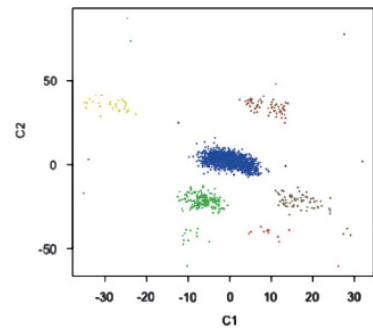

All populations, FCGR genotypes

indicated as separate colors

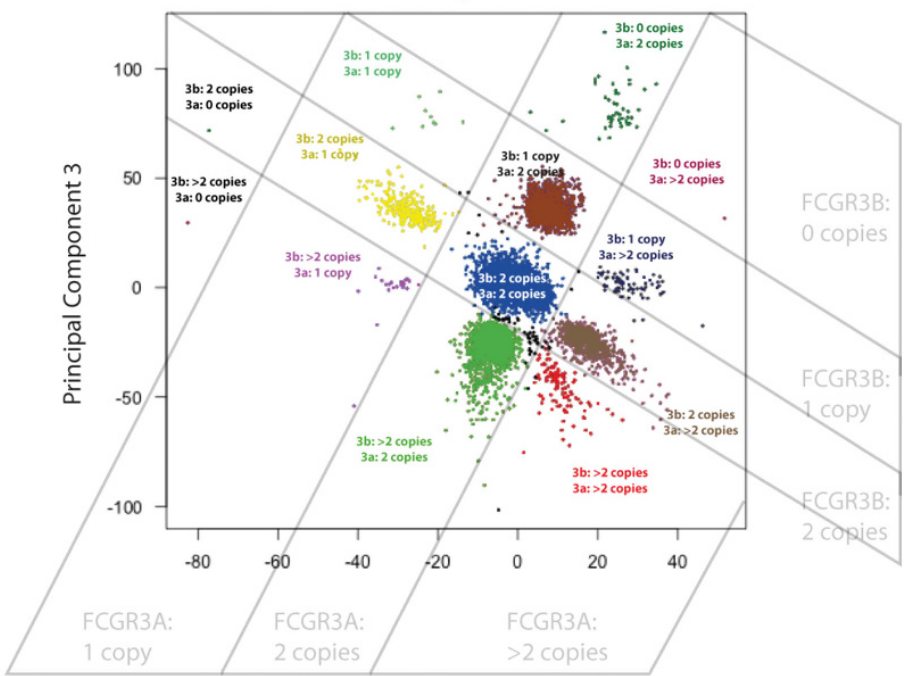

Italian population

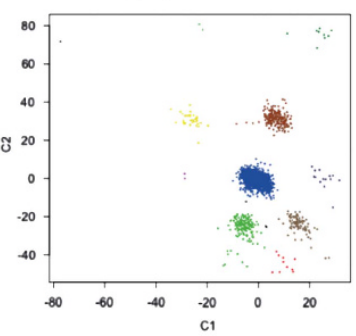

US population (I2B2 + Corrona)

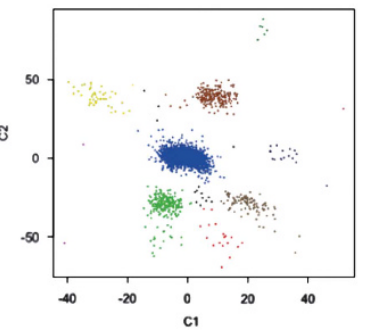

Polish population

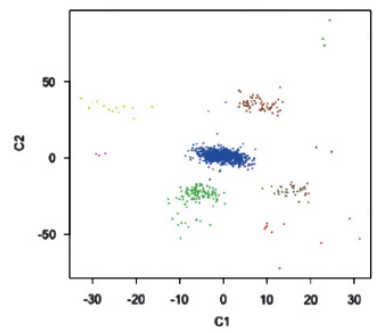

US population (Gregerson)

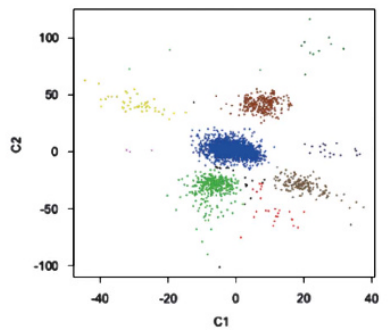

Figure 2 CNV frequency distributions of the FCGR3A and FCGR3B based on PC1 and PC3 of analysis of all 1159 single-nucleotide polymorphisms in the FCGR locus.

\section{Association of CNV in FCGR locus with RA, IBD and CeD}

We next investigated whether the CNVs in FCGR locus are associated with RA (including the CCP+ and CCP - groups), CeD and IBD. All analyses were performed separately per population and per disease (Supplementary Tables S7-S9) and then combined in a meta-analysis (Supplementary Table S10, Figure 3). We did not observe strong heterogeneity across populations (calculated by Breslow-Day test, Supplementary Table S11).

No significant association, in any group, was observed with deletions or duplications of FCGR3A block. Given the low frequency of FCGR3A deletions, it should be noted that our sample size only had sufficient power to determine strong effects of this rare variant (Supplementary Table S12).

In the FCGR3B gene, we observed associations of CCP - RA with duplication of the FCGR3B locus, where an extra copy of the FCGR3B block showed a susceptible effect in CCP - RA $(P=0.002$, OR 1.429,
95\% CI (1.146-1.782)). A similar, less significant, trend was observed in CeD analysis $(P=0.085, \mathrm{OR}=1.149,95 \% \mathrm{CI}(0.981-1.345))$.

Previous studies have indicated an association with deletion of the FCGR3B locus in CCP+ RA, although these results were inconsistent. ${ }^{16-18}$ In our analysis, we identified nominally significant association with deletion of the FCGR3B locus, and the trend of association was consistent with the previous observations $(P=0.023$, $\mathrm{OR}=1.229,95 \%$ CI (1.029-1.467); Figure 3 and Supplementary Table $\mathrm{S} 10)$. In the combined analysis of FCGR3A and FCGR3B blocks, trend of association of high CNV with CCP - RA was observed $(P=0.004$, $\mathrm{OR}=1.328,95 \%$ CI $(1.093-1.614))$.

Overall, only association of FCGR3B duplication with CCP - RA was significant after correction for multiple testing (Bonferroni; $P($ corr $)=0.02)$.

Although our method is capable of quantifying CNV in the FCGR locus properly, it is a fairly complicated procedure, requiring raw 
FCGR3A_low_vs_average

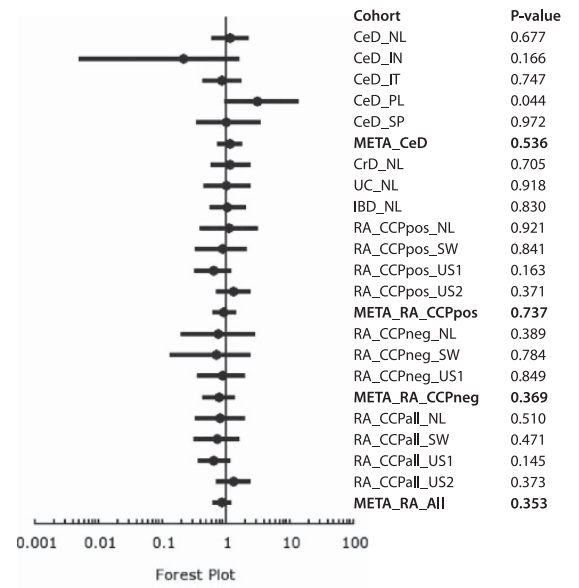

FCGR3B_low_vs_average

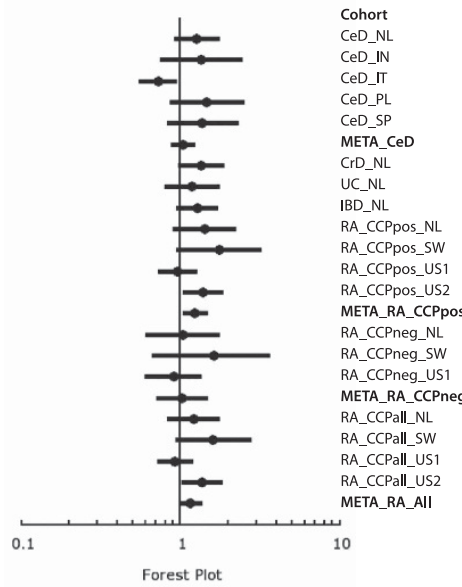

FCGR3AB_I ow_vs_average

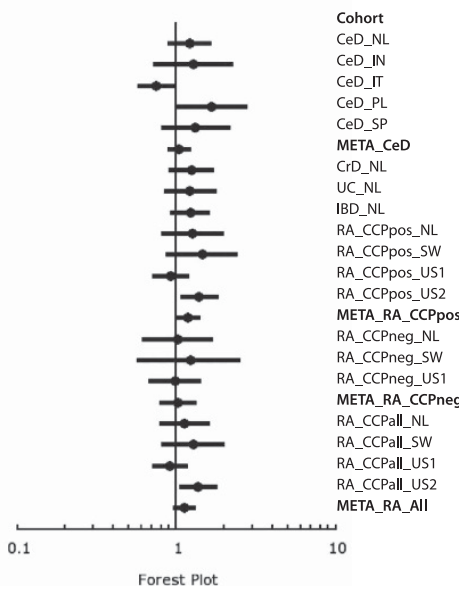

FCGR3A_high_vs_average

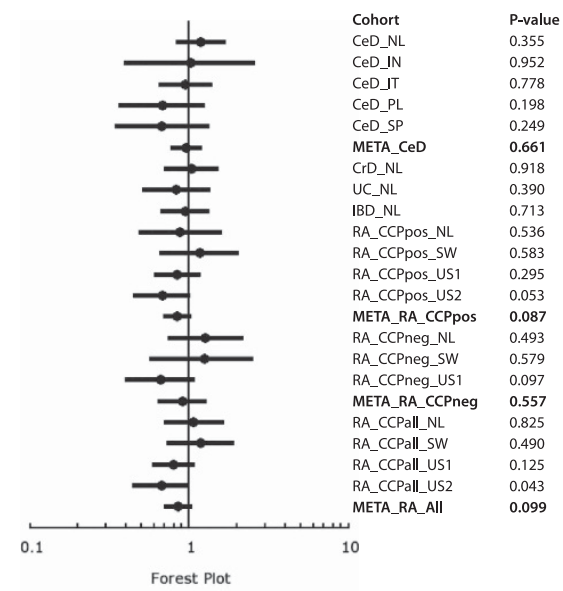

FCGR3B_high_vs_average

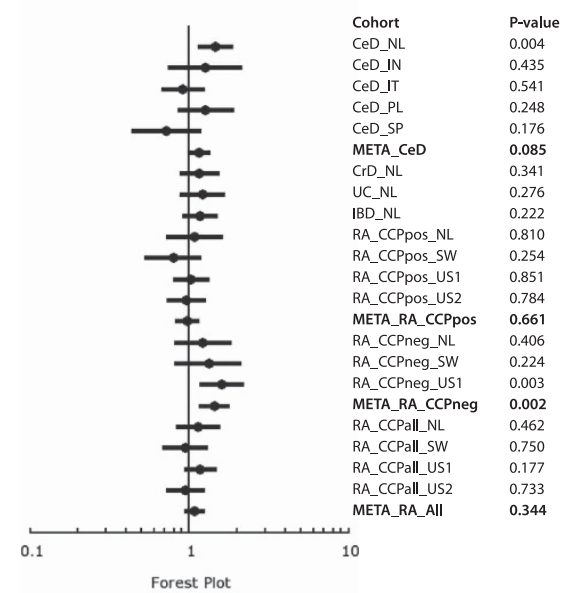

FCGR3AB_high_vs_average

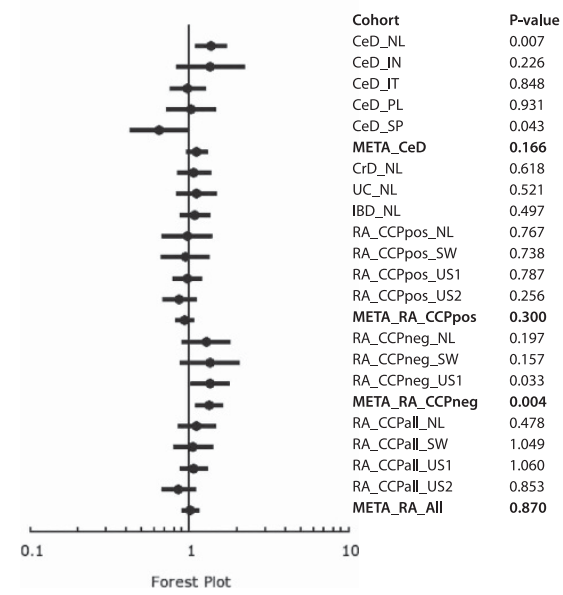

Figure 3 Meta-analysis of FCGR3A, FCGR3B and FCGR3A+FCGR3B blocks in RA, CD and IBD.

intensity data of both the FCGR locus and other autosomal regions in order to accurately correct for batch effects. A much more straightforward procedure would be possible if a normal bi-allelic SNP were in strong LD with the FCGR3A or FCGR3B loci. We therefore investigated whether any SNP in or around the locus could tag the
FCGR3A and/or FCGR3B CNVs. We found no SNP proxies to tag the CNV genotype ( $\max D^{\prime}=1, r^{2}<0.1$ ) (Supplementary Figure $S 4$ ); we therefore concluded that $\mathrm{CNV}$ estimation algorithms need to be applied to genotype the FCGR locus properly. Given the previous reports of association of SNPs in the FCGR locus to RA, we have also 
investigated the association of bi-allelic SNPs with RA, including the CCP+ and CCP - subgroup analysis. We looked for association in the locus chr1: 160,975,205-162143863 (from $500 \mathrm{~kb}$ left from start of FCGR2A till $500 \mathrm{~kb}$ right from end of FCGR3B gene). None of the SNPs were associated with RA with $P<0.003$ (including analyses in the $\mathrm{CCP}+$ and $\mathrm{CCP}$ - subgroups).

Expression of CD16 in relation to CNVs in FcyR locus. To identify the functional consequences of CNVs in FCGR3A and FCGR3B, we tested the expression of CD16 (Fc $\gamma$ RIII) receptor on neutrophils, monocytes, $\mathrm{B}$ and T cells. Despite previous studies ${ }^{17,40,41}$ reporting a correlation between neutrophil CD16b expression and FCGR3B copy number, we did not observe this correlation in our own samples (Supplementary Figure S5). However, we did observe a significant correlation of FCGR3A CNV and expression of CD16 on CD8+ T-cells ( $P$-value for correlation $P=0.0018$ ) (Figure 4). No correlation was observed for monocytes, $\mathrm{B}$ cells or $\mathrm{CD} 4+\mathrm{T}$ cells. We confirmed that the $\mathrm{Fc}$ receptor expressed on this subtype of $\mathrm{T}$ cells is the CD16a form (encoded by FCGR3A) by using PI-PLC, an enzyme that cleaves the GPI-linked form of CD16 (ie, CD16b, encoded by FCGR3B). This enzyme was not able to cleave CD16 from CD8+ T cells, although it was able to cleave CD16 from neutrophils, indicating that neutrophils carry CD16b and CD8+ T cells carry CD16a (Supplementary Figure S6). Further characterization of $\mathrm{CD} 8+\mathrm{CD} 16+\mathrm{T}$ cells indicated that they carry the $\alpha \beta$ T-cell receptor and have a terminally differentiated phenotype $^{42}$ based on the following characterization; CD45RA +CCR7 - CD27-CD28 - (data not shown).

\section{DISCUSSION}

The analysis of genetic association in SD loci is challenging due to their complex genetic structure. In this study, we have developed a method for $\mathrm{CNV}$ analysis in SD loci and applied it to the FCGR locus. Our method is based on PCA of raw intensity values of SNPs in CNV regions. We confirmed our results with three independent methods: segregation analysis in families, arrayCGH, and sequencing analysis. We found that CNVs were segregated in families, as expected, and there was a good correlation of the results from our method with those from NSG analysis. We also concluded that arrayCGH method

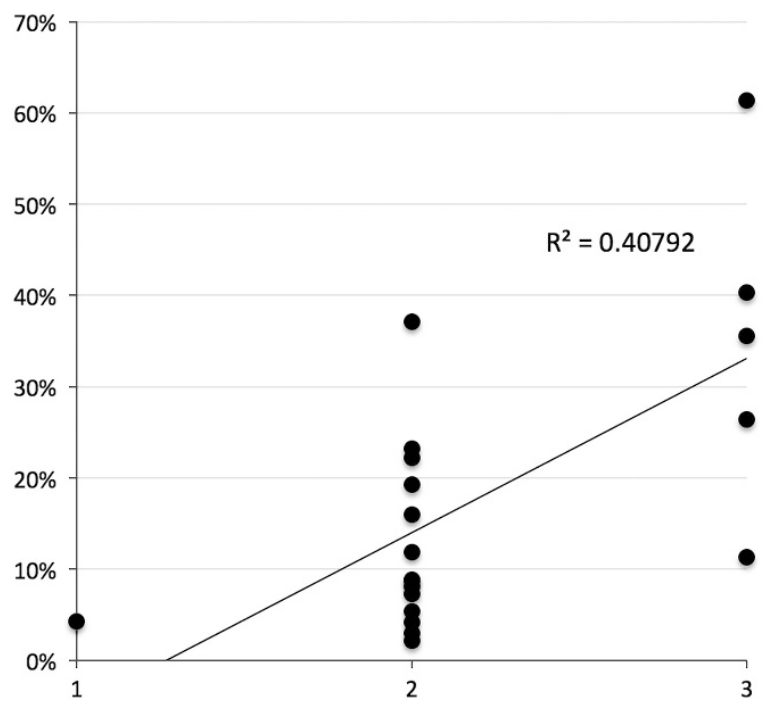

Figure 4 Correlation of FCGR3A CNV and expression of CD16 on CD8 cells. $X$-axis: different numbers of copies of FCGR3A. y Axis: percentage of CD16+CD8+ cells. is not accurate enough for fine-mapping CNVs in the FCGR locus, as it does not allow the specific identification of FCGR3A and FCGR3B CNVs. The CNV estimation from arrayCGH does, however, gives a good correlation with the total number of copies in both the FCGR3A and FCGR3B blocks. The boundaries of two CNV blocks identified in this study correspond approximately to the recent findings of breakpoints of FCGR3B deletion ( $24.5 \mathrm{~kb}$ region of identity in the FCGR2C and FCGR2B genes), identified by high-throughput sequencing analysis. $^{21}$

Association of low copies of FCGR3B with RA was recently suggested in a study of a cohort of 1115 cases and 654 healthy individuals $(P=0.028 ; \mathrm{OR}=1.50)$. This association appeared stronger in CCP+ individuals $(P=0.011 ; \quad \mathrm{OR}=1.61 ; 932 \mathrm{CCP}+$ cases included) ${ }^{17} \mathrm{~A}$ similar trend, although not significant, was observed by another meta-analysis that included a comparable number of samples $(P=0.15$; OR $=1.36) .{ }^{18}$ We applied our method to a large cohort of 17754 individuals with immune-mediated phenotypes and matched controls, including RA, CeD and IBD. As our study included 4578 RA patients (of which 3311 were CCP+ RA patients) and 5457 population-matched controls, we had sufficient power (Supplementary Table S12) to confirm the reported association mentioned above. We observed similar association with deletion in FCGR3B in the CCP+ subgroup of RA $(P=0.023, \mathrm{OR}=1.229)$, although this finding was not significant after correction for multiple testing. Similar results observed in three independent data sets suggest that association of deletion of FCGR3B in CCP+ RA patients is most likely a true-positive association and that the effect size of FCGR3B deletion on CCP+ RA is in the range of OR 1.2-1.5. We also identified an association in FCGR3B with the CCP - subgroup of RA, where high $\mathrm{CNV}$ in FCGR3B was associated with disease $(P=0.002$, OR 1.429). Overall, this indicates that CNVs in the FCGR3B locus show a different effect on the autoantibody-positive and -negative subgroups of RA. CCP can interact with FCGR3B leading to activation of various immune cells and are the most specific biomarkers for RA. It is now clear that the genetic contribution to CCP-positive and -negative disease is different. Our observation that the contribution of CNVs in the FCGR3B appears distinct for CCP-positive and -negative RA is in line with these findings that also indicate that CCP-positive and -negative disease have a different aetiology.

We did observe a significant positive correlation of FCGR3A CNV and the expression of CD16 on CD8+ T-cells, which has not been reported before. Intriguingly, this correlation is not present for other immune cells that express CD16a, indicating a cell-specific regulation of the expression of this receptor. The role of CD8+ T-cell immunity in RA is presently unclear, and it is not known whether CD16 expression on $\mathrm{CD} 8+\mathrm{T}$ cells is involved in the inflammatory process operative in RA. However, it would be interesting to know whether the CD16 expression of CD8 T cells differs between CD8+ $\mathrm{T}$ cells obtained from CCP-positive and -negative patients, as differential expression of CD16 in these two subgroups of RA would suggest a role for these cells in (CCP-positive) disease.

Overall, we have created a framework to identify deletions and duplications from intensity values of SNP genotypes on genome-wide association platforms. By confirming the results from our method with family segregation analysis, array CGH and sequencing analysis, we have shown that they are accurate and reliable and have identified a functional correlation between FCGR3A CNV with CD16 expression on CD8+ T cells. Our method can now be applied to other CNVs genotyped on the Immunochip platform or on any other dense SNP genotyping platform. 


\section{CONFLICT OF INTEREST}

The authors declare no conflict of interest.

\section{ACKNOWLEDGEMENTS}

We thank Jackie Senior and Kate Mc Intyre for critically reading the manuscript and Urmo Vosa, Patrick Deelen and Roan Kanninga for technical support. We thank all patients and control individuals for participating in this study. This study makes use of data generated by the Genome of the Netherlands Project. Samples were contributed by LifeLines (http://lifelines.nl/lifelinesresearch/general), The Leiden Longevity Study (http://www.healthy-ageing.nl; http://www.langleven.net), The Netherlands Twin Registry (NTR: http:// www.tweelingenregister.org), The Rotterdam studies, (http://www.erasmusepidemiology.nl/rotterdamstudy) and the Genetic Research in Isolated Populations programme (http://www.epib.nl/research/geneticepi/research. html\#gip). The sequencing was carried out in collaboration with the Beijing Institute for Genomics, China (BGI). This work was supported by the Centre for Translational Molecular Medicine (CTMM) (TRACER- 041-202/ 2009252), the Netherlands Organization for Health Research and Development (ZonMw), the European Community's Seventh Framework Programme (FP7/2007-2013, under grant agreement 259867), the Netherlands Organization for Scientific Research (NWO) (NWO-VICI grant 918.66.620 to CW, NWO-VENI grant 916.10.135 to LF, NWO-Rubicon grant to GT and NWO-BBMRI-NL 184021007), the Celiac Disease Consortium (an innovative cluster approved by the Netherlands Genomics Initiative and partly funded by the Dutch Government) (BSIK03009 to CW), the Dutch Digestive Diseases Foundation (MLDS WO11-30 to CW), the Dutch Arthritis Foundation (Reumafonds 11-1-101 to AZ) and a Horizon Breakthrough grant from the Netherlands Genomics Initiative (92519031 to LF).

1 Bailey JA, Eichler EE: Primate segmental duplications: crucibles of evolution, diversity and disease. Nat Rev Genet 2006; 7: 552-564.

2 Sharp AJ, Locke DP, McGrath SD et al: Segmental duplications and copy-number variation in the human genome. Am J Hum Genet 2005; 77: 78-88.

3 Dijstelbloem HM, Van de Winkel JG, Kallenberg CG: Inflammation in autoimmunity: receptors for IgG revisited. Trends Immunol 2001; 22: 510-516.

4 Fanciulli M, Vyse TJ, Aitman TJ: Copy number variation of Fc gamma receptor genes and disease predisposition. Cytogenet Genome Res 2008; 123: 161-168.

5 Von Bubnoff D, Novak N, Kraft S, Bieber T: The central role of FcepsilonRI in allergy. Clin Exp Dermatol 2003; 28: 184-187.

6 Indik ZK, Park JG, Hunter S, Schreiber AD: The molecular dissection of Fc gamma receptor mediated phagocytosis. Blood 1995; 86: 4389-4399.

7 Huizinga TW, Dolman KM, Van der Linden NJ et al: Phosphatidylinositol-linked FcRIII mediates exocytosis of neutrophil granule proteins, but does not mediate initiation of the respiratory burst. J Immunol 1990; 144: 1432-1437.

8 Gessner JE, Heiken H, Tamm a, Schmidt RE: The IgG Fc receptor family. Ann Hematol 1998; 76: 231-248.

9 Jostins L, Ripke S, Weersma RK et al: Host-microbe interactions have shaped the genetic architecture of inflammatory bowel disease. Nature 2012; 491: 119-124.

10 Anderson C, Boucher G, Lees CW et al: Meta-analysis identifies 29 additional ulcerative colitis risk loci, increasing the number of confirmed associations to 47. Nat Genet 2011; 43: 246-252.

11 Harley JB, Alarcón-Riquelme ME, Criswell LA et al: Genome-wide association scan in women with systemic lupus erythematosus identifies susceptibility variants in ITGAM, PXK, KIAA1542 and other loci. Nat Genet 2008; 40: 204-210.

12 Raychaudhuri S, Thomson BP, Remmers EF et al: Genetic variants at CD28, PRDM1 and CD2/CD58 are associated with rheumatoid arthritis risk. Nat Genet 2009; 41: 1313-1318.

13 Nossent JC, Rischmueller M, Lester S: Low copy number of the Fc- $\gamma$ receptor $3 B$ gene FCGR3B is a risk factor for primary Sjogren's syndrome. J Rheumatol 2012; 39: 2142-2147.

14 Niederer $\mathrm{H}$ a, Willcocks LC, Rayner TF et al: Copy number, linkage disequilibrium and disease association in the FCGR locus. Hum Mol Genet 2010; 19: 3282-3294.

15 Fanciulli M, Norsworthy PJ, Petretto E et al: FCGR3B copy number variation is associated with susceptibility to systemic, but not organ-specific, autoimmunity. Nat Genet 2007; 39: 721-723.
16 Thabet MM, Huizinga TWJ, Marques RB et al: Contribution of Fcgamma receptor IIIA gene $158 \mathrm{~V} / \mathrm{F}$ polymorphism and copy number variation to the risk of ACPA-positive rheumatoid arthritis. Ann Rheum Dis 2009; 68: 1775-1780.

17 Robinson JI, Carr IM, Cooper DL et al: Confirmation of association of FCGR3B but not FCGR3A copy number with susceptibility to autoantibody positive rheumatoid arthritis. Hum Mutat 2012; 33: 741-749.

18 McKinney C, Merriman TR: Meta-analysis confirms a role for deletion in FCGR3B in autoimmune phenotypes. Hum Mol Genet 2012; 21: 2370-2376.

19 Aitman TJ, Dong R, Vyse TJ et al: Copy number polymorphism in Fcgr3 predisposes to glomerulonephritis in rats and humans. Nature 2006; 439: 851-855.

20 Niederer HA, Clatworthy MR, Willcocks LC, Smith KGC: FC $\gamma$ RIIB, FC $\gamma$ RIIIB, and systemic lupus erythematosus. Ann N Y Acad Sci 2010; 1183: 69-88.

21 Mueller M, Barros P, Witherden AS et al: Genomic pathology of SLE-associated copynumber variation at the FCGR2C/FCGR3B/FCGR2B locus. Am J Hum Genet 2013; 92: 28-40.

22 Craddock N, Hurles ME, Cardin N et al: Genome-wide association study of CNVs in 16,000 cases of eight common diseases and 3,000 shared controls. Nature 2010; 464: 713-720.

23 Breunis WB, Van Mirre E, Geissler J et al: Copy number variation at the FCGR locus includes FCGR3A, FCGR2C and FCGR3B but not FCGR2A and FCGR2B. Hum Mutat 2009; 30: E640-E650.

24 Hollox EJ, Detering J-C, Dehnugara T: An integrated approach for measuring copy number variation at the FCGR3 (CD16) locus. Hum Mutat 2009; 30: 477-484.

25 Eyre S, Bowes J, Diogo D et al: High-density genetic mapping identifies new susceptibility loci for rheumatoid arthritis. Nat Genet 2012; 44: 1336-1340.

26 Trynka G, Hunt KA, Bockett NA et al: Dense genotyping identifies and localizes multiple common and rare variant association signals in celiac disease. Nat Genet 2011; 43: 1193-1201.

27 Cooper JD, Simmonds MJ, Walker NM et al: Seven newly identified loci for autoimmune thyroid disease. Hum Mol Genet 2012; 21: 5202-5208.

28 Juran BD, Hirschfield GM, Invernizzi P et al: Immunochip analyses identify a novel risk locus for primary biliary cirrhosis at $13 q 14$, multiple independent associations at four established risk loci and epistasis between $1 \mathrm{p} 31$ and $7 \mathrm{q} 32$ risk variants. Hum Mol Genet 2012; 21: 5209-5221.

29 Wang K, Li M, Hadley D et al: PennCNV: an integrated hidden Markov model designed for high-resolution copy number variation detection in whole-genome SNP genotyping data. Genome Res 2007; 17: 1665-1674.

30 Colella S, Yau C, Taylor JM et al: QuantiSNP: an Objective Bayes Hidden-Markov Model to detect and accurately map copy number variation using SNP genotyping data. Nucleic Acids Res 2007; 35: 2013-2025.

31 Franke L, De Kovel CGF, Aulchenko YS et al: Detection, imputation, and association analysis of small deletions and null alleles on oligonucleotide arrays. Am J Hum Genet 2008; 82: 1316-1333.

32 Cardin N, Holmes C, Donnelly P, Marchini J: Bayesian hierarchical mixture modeling to assign copy number from a targeted CNV array. Genet Epidemiol 2011; 35: 536-548.

33 Barnes C, Plagnol V, Fitzgerald $T$ et al: A robust statistical method for casecontrol association testing with copy number variation. Nat Genet 2008; 40: 1245-1252.

34 Pinto D, Darvishi K, Shi $X$ et al: Comprehensive assessment of array-based platforms and calling algorithms for detection of copy number variants. Nat Biotechnol 2011; 29: 512-520.

35 Korn JM, Kuruvilla FG, McCarroll SA et al: Integrated genotype calling and association analysis of SNPs, common copy number polymorphisms and rare CNVs. Nat Genet 2008; 40: 1253-1260.

36 Cooper GM, Zerr T, Kidd JM, Eichler EE, Nickerson DA: Systematic assessment of copy number variant detection via genome-wide SNP genotyping. Nat Genet 2008; 40: 1199-1203.

37 Kumasaka N, Fujisawa H, Hosono N et al: PlatinumCNV: a Bayesian Gaussian mixture model for genotyping copy number polymorphisms using SNP array signal intensity data. Genet Epidemiol 2011; 35: 831-844.

38 Boomsma DI, Wijmenga C, Slagboom EP et al: The Genome of the Netherlands: design, and project goals. Eur J Hum Genet 2014; 22: 221-227.

39 Barrett JC, Fry B, Maller J, Daly MJ: Haploview: analysis and visualization of LD and haplotype maps. Bioinformatics 2005; 21: 263-265.

40 Willcocks LC, Lyons PA, Clatworthy MR et al: Copy number of FCGR3B, which is associated with systemic lupus erythematosus, correlates with protein expression and immune complex uptake. J Exp Med 2008; 205: 1573-1582.

41 Morris DL, Roberts AL, Witherden AS et al: Evidence for both copy number and allelic (NA1/NA2) risk at the FCGR3B locus in systemic lupus erythematosus. Eur J Hum Genet 2010; 18: 1027-1031.

42 Björkström NK, Gonzalez VD, Malmberg K-J et al: Elevated numbers of Fc gamma RIIIA $+(\mathrm{CD} 16+)$ effector CD8 T cells with NK cell-like function in chronic hepatitis C virus infection. J Immunol 2008; 181: 4219-4228.

Supplementary Information accompanies this paper on European Journal of Human Genetics website (http://www.nature.com/ejhg) 